05

\title{
Динамика решетки магнитных нанодиполей с кубической анизотропией
}

\author{
() А.М. Шутый, Д.И. Семенцов \\ Ульяновский государственный университет, \\ Ульяновск, Россия \\ E-mail: shuty@mail.ru \\ (Поступила в Редакцию 24 января 2017 г.)
}

Компьютерным моделированием исследованы квадратные $5 \times 5$ решетки магнитных диполей с кубической кристаллографической анизотропией. Выявлены условия реализации произвольных ориентационных конфигураций решеток, каждая из которых характеризуется определенным откликом на воздействие внешнего магнитного импульса, а также устанавливаемым режимом колебаний суммарного магнитного момента под действием переменного поля. Обнаружены регулярные колебательные режимы с удвоенной частотой, квазипериодические и хаотические режимы. Исследована зависимость отклика систем от параметров импульса магнитного поля.

Работа выполнена при поддержке Министерства образования и науки РФ в рамках Государственного задания № 3.6825.2017/БЧ и проекта 14.Z50.31.0015.

DOI: 10.21883/FTT.2017.09.44840.011

\section{1. Введение}

В последние годы ведется активное изучение создаваемых нанотехнологиями магнитных сверхструктур и ансамблей магнитных частиц [1-7]. Среди таких структур особый интерес представляют состоящие из однодоменных нанодиполей одномерные и двумерные структуры, в частности, магнитоупорядоченные квадратные решетки. Подобные структуры могут быть сформированы, например, на основе наночастиц ферромагнитных металлов [8]. Основной вклад во взаимодействие магнитных моментов в таких решетках вносит диполь-дипольное взаимодействие $[8,9]$. В работах [10-13] рассмотрены равновесные состояния в линейных цепочках и квадратных решетках магнитных моментов нанодиполей и динамические режимы, возникающие в процессе их перемагничивания во внешнем статическом магнитном поле. Дискретность структур приводит к существенным отличиям статических и динамических свойств подобных решеток от свойств макроскопических монодоменных объектов. К таким отличиям, в частности, могут быть отнесены бистабильные состояния систем, обусловленные наличием различных ориентационных конфигураций, отличающихся суммарным магнитным моментом систем, а также осуществление управляемых переходов между данными конфигурациями и возникновение динамических колебательных режимов магнитного момента системы при их перемагничивании.

Ансамбли ферромагнитных частиц могут быть использованы в качестве носителей информации. В частности, в работе [14] рассматривается трехмерная структура диполей с кристаллографической анизотропией. При этом запись информации заключается в изменении равновесной конфигурации системы за счет воздействия на нее радиоимпульсов поля большой амплитуды, а считы- вание обеспечивается возбуждением возникшей конфигурации маломощным радиоимпульсом магнитного поля на частоте ферромагнитного резонанса и сканированием частоты отклика дипольной системы.

В настоящей работе на основе компьютерного моделирования исследуются статические и динамические режимы дипольных решеток, состоящих из однодоменных магнитных наночастиц, обладающих кубической кристаллографической анизотропией. Рассмотрены равновесные конфигурации данных решеток, колебательные режимы, вызванные воздействием на них переменного магнитного поля, а также особенности их отклика на импульс внешнего поля. Основное внимание уделено системам, в которых кристаллографическая анизотропия является определяющей при установлении равновесной ориентации каждой из входящих в решетку наночастиц.

\section{2. Исходные уравнения}

Рассмотрим квадратную решетку из системы $5 \times 5$ взаимодействующих друг с другом магнитных нанодиполей с одинаковыми по величине магнитными моментами $\left|\mathbf{m}_{i}\right|=m$. Энергию $i$-го нанодиполя запишем в виде суммы зеемановской энергии во внешнем магнитном поле $\mathbf{H}$, энергии кубической анизотропии дипольной наночастицы и энергии диполь-дипольного взаимодействия

$$
W_{i}=-\mathbf{m}_{i} \mathbf{H}+W_{a}\left(\mathbf{m}_{i}\right)+\sum_{n} W_{d}\left(\mathbf{m}_{i}, \mathbf{m}_{n}\right)
$$

Здесь внешнее магнитное поле является суммой статического и высокочастотного полей $\mathbf{H}=\mathbf{H}_{c}+\mathbf{H}(t)$; энергия кубической анизотропии в случае, когда оси $X$, $Y, Z$ направлены вдоль трех кристаллографических 
осей $\langle 100\rangle$ наночастицы, имеет вид

$$
W_{a}=\frac{V K_{1}}{m^{4}}\left(m_{i x}^{2} m_{i y}^{2}+m_{i y}^{2} m_{i z}^{2}+m_{i z}^{2} m_{i x}^{2}\right),
$$

где $K_{1}$ - константа кубической анизотропии материала наночастицы; $V$ - объем наночастицы. Будем считать ось $X$ перпендикулярной плоскости решетки нанодиполей, а две другие оси - параллельными сторонам этой решетки. Энергию диполь-дипольного взаимодействия представим в виде

$$
W_{d}\left(\mathbf{m}_{i}\right)=\sum_{n \neq i}\left(\frac{\mathbf{m}_{i} \mathbf{m}_{n} r_{i n}^{2}-3\left(\mathbf{m}_{i} \mathbf{r}_{i n}\right)\left(\mathbf{m}_{n} \mathbf{r}_{i n}\right)}{r_{i n}^{5}}\right),
$$

где $\mathbf{r}_{i n}$ и $r_{i n}-$ радиус-вектор и расстояние между $i$-ым и $n$-ым диполями.

Динамика каждого из магнитных моментов описывается уравнением Ландау-Лифшица с релаксационным членом в форме Гильберта [15]:

$$
\frac{\partial \mathbf{m}_{i}}{\partial t}=-\gamma \mathbf{m}_{i} \mathbf{H}_{i}^{e f}-\frac{\alpha}{m_{i}} \mathbf{m}_{i} \frac{\partial \mathbf{m}_{i}}{\partial t},
$$

где $\gamma-$ гиромагнитное отношение, $\alpha-$ одинаковый для всех частиц параметр диссипации. Эффективное магнитное поле, создаваемое в месте расположения $i$-го диполя остальными диполями и внешним полем $\mathbf{H}$, имеет вид:

$$
\mathbf{H}_{i}^{e f}=-\frac{\partial W_{i}}{\partial \mathbf{m}_{i}}=\mathbf{H}+\mathbf{H}_{a i}+\sum_{n \neq i} \frac{3\left(\mathbf{m}_{n} \mathbf{r}_{i n}\right) \mathbf{r}_{i n}-\mathbf{m}_{n} r_{i n}^{2}}{r_{i n}^{5}} .
$$

Для поля кубической анизотропии каждая из трех компонент $(v \equiv x, y, z)$ определяется выражением:

$$
H_{a i v}=-\frac{2 V K_{1}}{m} \mu_{i v}\left(1-\mu_{i v}^{2}\right),
$$

где $\boldsymbol{\mu}_{i}=\mathbf{m}_{i} / m$. Далее перейдем к безразмерным параметрам: $\mathbf{e}_{i n}=\mathbf{r}_{i n} / r_{i n}, \tau=\left(m \gamma / d^{3}\right) t$, где $d-$ расстояние между центрами ближайших наночастиц; $l_{i n}=r_{i n} / d$. $\mathrm{B}$ безразмерных параметрах уравнения (5) принимают вид:

где

$$
\frac{\partial \boldsymbol{\mu}_{i}}{\partial \tau}=-\boldsymbol{\mu}_{i} \mathbf{h}_{i}^{e f}-\alpha \boldsymbol{\mu}_{i} \frac{\partial \boldsymbol{\mu}_{i}}{\partial \tau},
$$

$$
\mathbf{h}_{i}^{e f}=\mathbf{h}+\mathbf{h}_{a i}+\sum_{n \neq i}\left[\frac{3\left(\boldsymbol{\mu}_{n} \mathbf{e}_{i n}\right) \mathbf{e}_{i n}-\boldsymbol{\mu}_{n}}{l_{i n}^{3}}\right] .
$$

При этом внешнее поле $-\mathbf{h}=\mathbf{H} d^{3} / m$, а поле кубической анизотропии:

$$
h_{a i j}=-2 k_{1} \mu_{i j}\left(1-\mu_{i j}^{2}\right),
$$

где $k_{1}=K_{1} V d^{3} / m^{2}-$ константа анизотропии. Для дальнейшего анализа векторное уравнение (7) представляется тремя скалярными уравнениями. Так, для $x$-компоненты получаем:

$$
\begin{gathered}
\left(1+\alpha^{2}\right) \frac{\partial \mu_{i x}}{\partial \tau}=\left(\mu_{i z}+\alpha \mu_{i x} \mu_{i y}\right) h_{i y}^{e f}-\left(\mu_{i y}-\alpha \mu_{i z} \mu_{i x}\right) h_{i z}^{e f} \\
-\alpha\left(1-\mu_{i x}^{2}\right) h_{i x}^{e f} .
\end{gathered}
$$

Уравнения для остальных компонент величины $\partial \boldsymbol{\mu}_{i} / \partial \tau$ имеют аналогичный вид и могут быть получены циклической перестановкой индексов $x, y, z$.

\section{3. Равновесные ориентационные конфигурации}

Равновесные ориентации отдельных диполей квадратных решеток $5 \times 5$ и динамические режимы решетки в целом определялись с учетом связи всех элементов

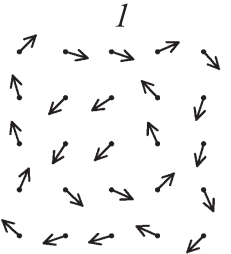

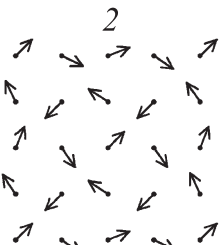

$$
\begin{aligned}
& y \rightarrow \stackrel{3}{\rightarrow}>\pi \\
& \llcorner\ll \leftarrow \\
& v \pi \rightarrow y \pi \\
& \downarrow \pi<< \\
& v \rightarrow \rightarrow \rightarrow \pi \\
& \leftarrow \leftarrow \stackrel{4}{\leftarrow} \leftarrow \leftarrow \\
& \downarrow \rightarrow \stackrel{5}{\rightarrow} \rightarrow 1 \\
& \downarrow \rightarrow \rightarrow \rightarrow 1 \\
& \rightarrow \rightarrow \rightarrow \rightarrow \rightarrow \\
& \uparrow \rightarrow \rightarrow \rightarrow \downarrow \\
& \uparrow \rightarrow \rightarrow \rightarrow \downarrow \\
& 6 \\
& \begin{array}{l}
\rightarrow \rightarrow \rightarrow \rightarrow \\
\leftarrow \leftarrow \leftarrow \leftarrow \leftarrow
\end{array} \\
& \rightarrow \rightarrow \rightarrow \rightarrow \rightarrow \\
& \leftarrow \leftarrow \leftarrow \leftarrow \leftarrow
\end{aligned}
$$

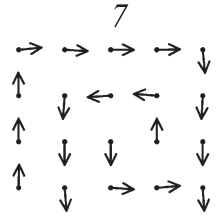

$$
\begin{aligned}
& \begin{array}{l}
\stackrel{8}{\downarrow} \rightarrow \rightarrow \uparrow \\
\downarrow \rightarrow \rightarrow \rightarrow \uparrow \\
\rightarrow \rightarrow \rightarrow \rightarrow \rightarrow \\
\uparrow \rightarrow \rightarrow \rightarrow \downarrow \\
\uparrow \rightarrow \rightarrow \rightarrow
\end{array} \\
& \rightarrow \rightarrow \rightarrow \rightarrow \rightarrow \\
& \rightarrow \rightarrow \rightarrow \rightarrow \rightarrow \\
& \rightarrow \rightarrow \rightarrow \rightarrow \rightarrow \\
& \rightarrow \rightarrow \rightarrow \rightarrow \rightarrow \\
& \rightarrow \rightarrow \rightarrow \rightarrow \rightarrow \\
& 9 \\
& \leftarrow \leftarrow \leftarrow \leftarrow \leftarrow \\
& \downarrow \uparrow \rightarrow \rightarrow \downarrow \\
& \downarrow \uparrow \leftarrow \leftarrow \downarrow \\
& \rightarrow \rightarrow \rightarrow \rightarrow \downarrow \\
& \leftarrow \leftarrow \leftarrow \leftarrow \downarrow
\end{aligned}
$$

Рис. 1. Равновесные конфигурации решеток нанодиполей в отсутствие внешнего поля при $k_{1}=0$ (конфигурации $1-3$ ), $k_{1}=-0.5,-1.5,-2$ (конфигурации $\left.4-6\right), k_{1}=-10$ (конфигурации 7-9).

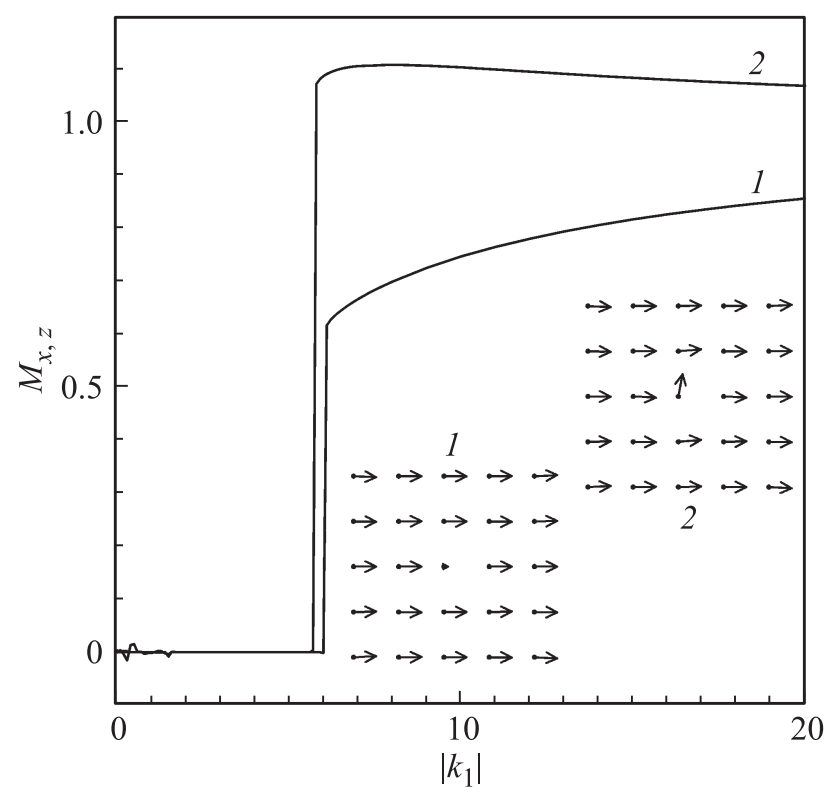

Рис. 2. Зависимость равновесных $x$ - и $z$-компонент (кривые 1 и 2) суммарного магнитного момента от величины константы анизотропии; в исходном состоянии центральный диполь ориентирован по оси $X$ или $Z$ (кривые 1,2 ), остальные диполи по оси $Y$; приведены конфигурации с $k_{1}=-10$. 

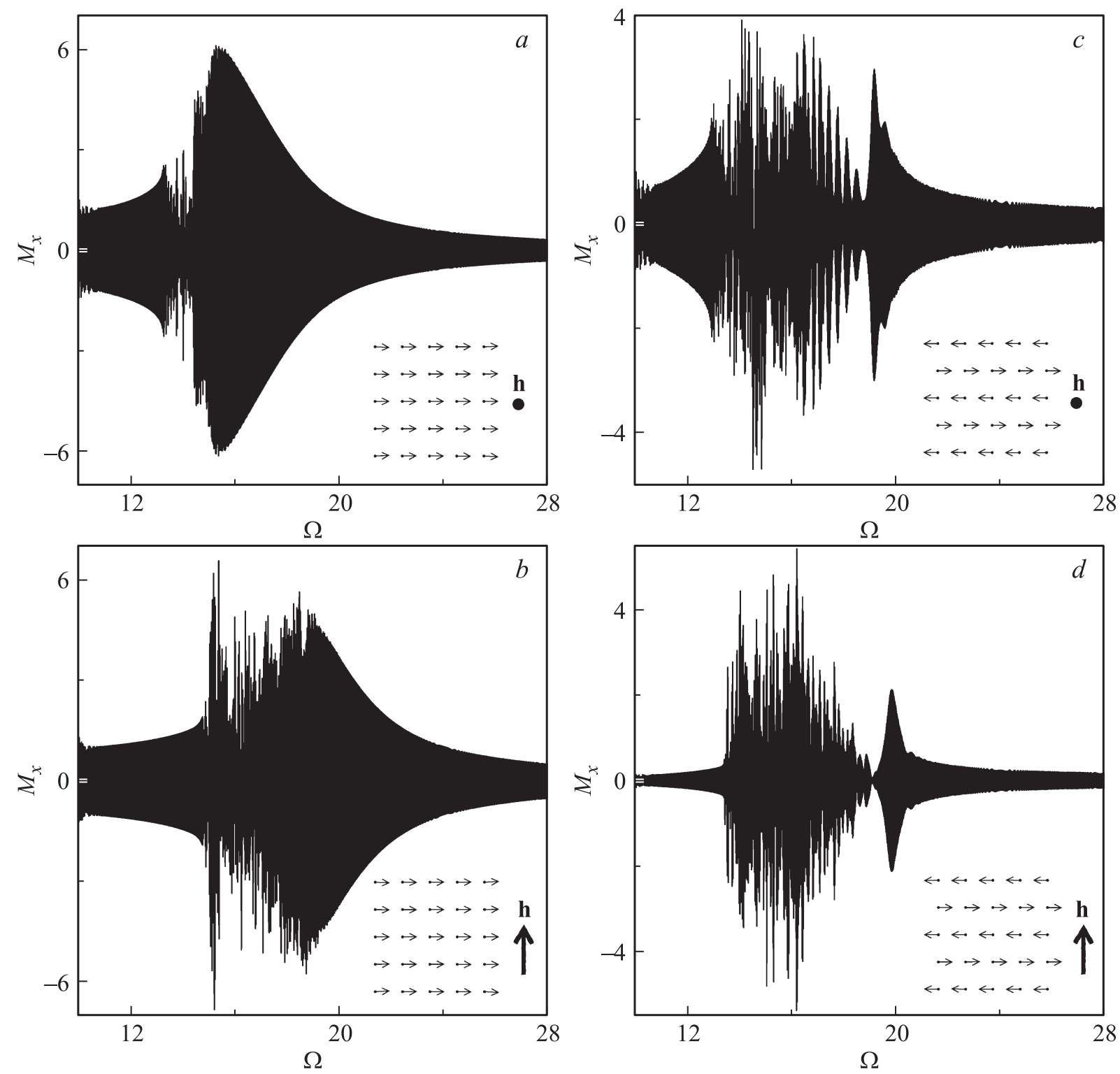

Рис. 3. Зависимость амплитуды $x$-компоненты магнитного момента решетки от частоты переменного поля при квазистатическом ее увеличении для двух равновесных конфигураций решетки при $k_{1}=-10$ и направлениях переменного поля по оси $X(a, c)$ и по оси $Z(b, d)$ с амплитудой $h_{0}=1$.

ансамбля друг с другом на основе численного решения приведенных выше уравнений методом Рунге-Кутта четвертого порядка. При отсутствии внешних полей равновесные ориентационные конфигурации находились заданием произвольных начальных состояний магнитных моментов всех диполей, из которых система приходит к стационарному состоянию согласно приведенным уравнениям движения.

На рис. 1 приведены равновесные конфигурации указанных решеток, полученные в отсутствие внешнего статического поля при различных значениях константы кубической анизотропии: $k_{1}=0$ (конфигурации $1-3$ ), $k_{1}=-0.5,-1.5,-2(4-6)$ и $k_{1}=-10(7-9)$. Конфигурации 1-3 отвечают случаю изотропных наночастиц и отличаются значением суммарного магнитного мо- мента $\mathbf{M}=\sum \boldsymbol{\mu}_{i}$. Каждая из указанных конфигураций устанавливается в соответствии с исходным состоянием системы. С ростом кристаллографической анизотропии число возможных устанавливаемых равновесных конфигураций увеличивается. Так как нами рассматривается анизотропия типа [100], направление ориентации магнитных моментов наночастиц приближается к плоскостным осям системы $Y$ или $Z$. Установлено, что для $\left|k_{1}\right|>10$ конфигурации практически не отличаются от представленных случаев (7-9), при этом равновесными являются состояния с любой ориентацией каждого из магнитных моментов решетки, совпадающей с направлением одной из кристаллографических осей.

На рис. 2 представлена зависимость от константы анизотропии компонент суммарного магнитного момента 
решетки в равновесном состоянии, когда все диполи, кроме центрального, ориентированы в плоскости решетки вдоль оси $Y$, а центральный диполь в исходном состоянии ориентирован локальным внешним полем по оси $X$ (кривая 1 , которой на графике соответствует компонента $M_{x}$ ) и по оси $Z$ (кривая 2, которой соответствует компонента $M_{z}$ ). Из рисунка видно, что в случае систем с $\left|k_{1}\right| \leq 6$ при выключении внешнего локального поля центральный диполь оказывается ориентированным, как и соседние диполи, по оси $Y$. В случае же $\left|k_{1}\right|>6$ центральный диполь остается ориентированным преимущественно по осям $X(1)$ и $Z(2)$ - на рисунке приведены соответствующие конфигурации систем при $k_{1}=-10$. В обоих случаях локальное поле, выводящее центральный диполь на указанные направления, должно иметь величину $h_{c}>8$.

Таким образом, для систем с $\left|k_{1}\right|>6$ (или с $\left.\left|K_{1}\right|>6 m^{2} / V d^{3}\right)$ диполь-дипольное взаимодействие слабо влияет на равновесные конфигурации, и все магнитные моменты решетки ориентируются вблизи трех кристаллографических направлений, выбор между которыми является достаточно произвольным. При этом внешним локальным полем может быть выстроена любая конфигурация дипольной системы, которая практически не изменится после выключения внешнего поля. В частности, может быть реализована конфигурация с одним диполем, ориентированным в направлении, близком к перпендикулярному к плоскости системы, тогда как все другие диполи лежат в плоскости.

Различным конфигурациям решетки отвечают различные ее отклики на воздействие импульса внешнего магнитного поля. Различными являются также стационарные прецессионные режимы суммарного магнитного момента при действии на систему диполей внешнего переменного поля. Все это дает возможность использовать рассматриваемые решетки магнитных диполей в качестве систем для записи информации и ее считывания с помощью слабых импульсов и внешних переменных полей.

\section{4. Динамика решеток с различной конфигурацией}

С ростом числа ориентированных вдоль нормали к плоскости решетки (т.е. поднятых) диполей не только увеличивается амплитуда, вызванных внешним полем, колебаний, но и существенно меняется вид колебаний. При этом устанавливаемые динамические режимы зависят от расположения в системе поднятых диполей, в частности, от расстояния между ними, что объясняется взаимодействием между данными диполями, а также между ними и остальными диполями решетки. Рассмотрим систему, в которой два диполя ориентированы вблизи нормали к плоскости решетки, а другие диполи лежат в плоскости и ориентированы вдоль оси $Y$. Исследуем колебательные режимы магнитного момента такой системы, устанавливаемые переменным магнитным полем: $\mathbf{h}(\tau)=\mathbf{h}_{0} \sin (\Omega \tau)$, где $\Omega$ - обезразмеренная частота, связанная с реальной частотой поля $\omega$ соотношением $\Omega=\left(d^{3} / m \gamma\right) \omega$. Резонансная частота изолированных магнитных моментов $\omega_{0}=\gamma H$, и

$$
\Omega_{0}=\frac{d^{3} \omega_{0}}{m \mathcal{\gamma}}=h .
$$

Взаимодействие между диполями изменяет частоту резонанса, при этом зависимость амплитуды прецессии от частоты поля становится нелинейной.

На рис. 3 приведена зависимость амплитуды $x$-компоненты магнитного момента дипольной решетки от частоты переменного поля при квазистатическом ее увеличении. Рассматривались две исходные конфигурации решеток с $k_{1}=-10$, приведенные на рисунках, и два направления линейно поляризованного переменного поля: по оси $X(a, c)$ и по оси $Z(b, d)$ с амплитудой $h_{0}=1$. При отсутствии взаимодействия между диполями резонансная частота определялась бы только полем

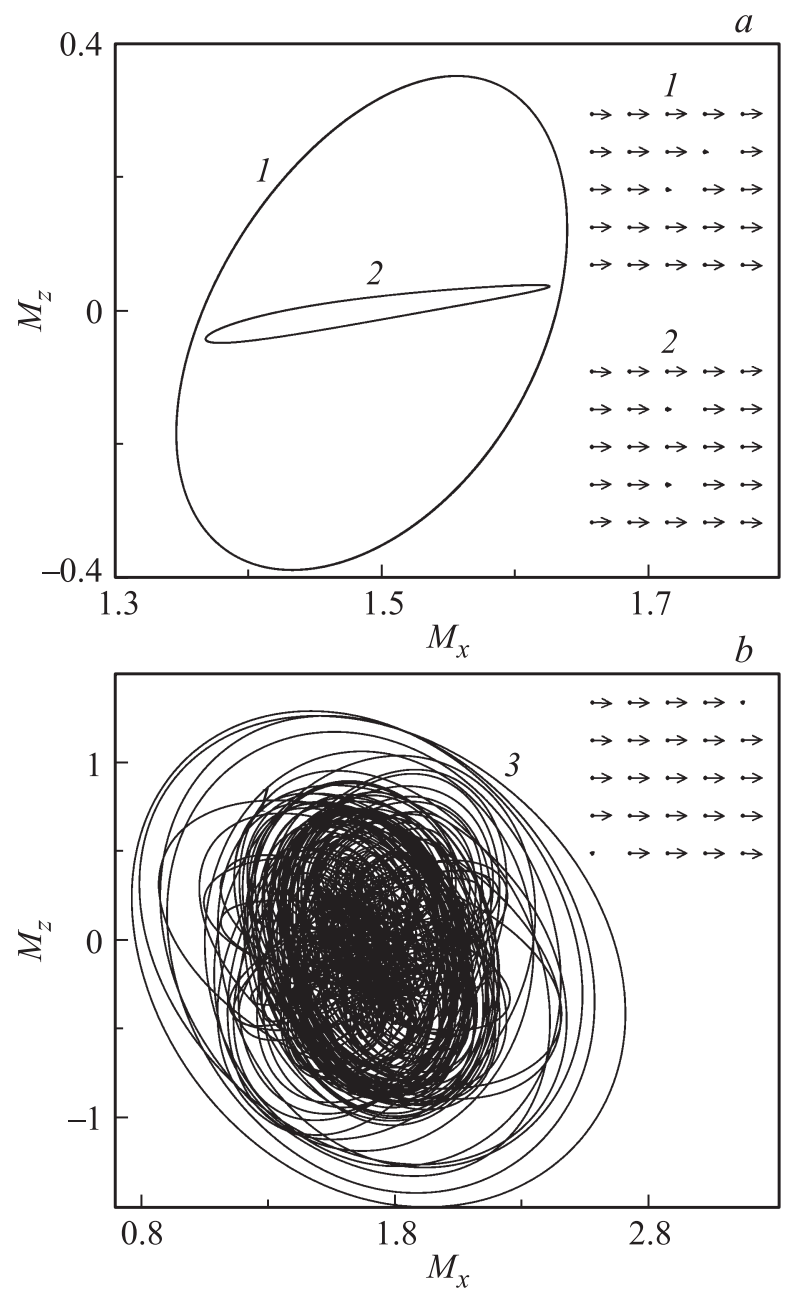

Рис. 4. Проекции на плоскость $Z X$ траекторий установившихся колебательных режимов магнитного момента систем с $k_{1}=-10$ и конфигурации решеток при линейно поляризованном вдоль оси $Y$ переменном поле с $\Omega=20$ и $h_{0 y}=1$; параметр диссипации $\alpha=0.01$. 
анизотропии $\Omega=2\left|k_{1}\right|$. В случае сонаправленных магнитных диполей $(a, b)$ резонансная частота смещается в область меньших значений. В наибольшей степени это проявляется при плоскостном направлении переменного поля, где $\Omega \approx 16$. В случае, когда соседние ряды диполей имеют противоположное направление $(c, d)$, максимум амплитуды регулярного колебательного режима близок к значению $\Omega \approx 20$, значительная область частот относится к хаотической динамике. Таким образом, далее в расчетах систем с преобладанием поля анизотропии в качестве частоты стационарного переменного поля, а также в качестве несущей частоты импульса магнитного поля будет браться частота $\Omega=2 k_{1}$.

На рис. 4 приведены проекции на плоскость $Z X$ траекторий установившихся колебательных режимов магнитного момента систем с $k_{1}=-10$ при действии линейно поляризованного по оси $Y$ переменного поля с частотой $\Omega=20$ и амплитудой $h_{0 y}=1$. Параметр диссипации
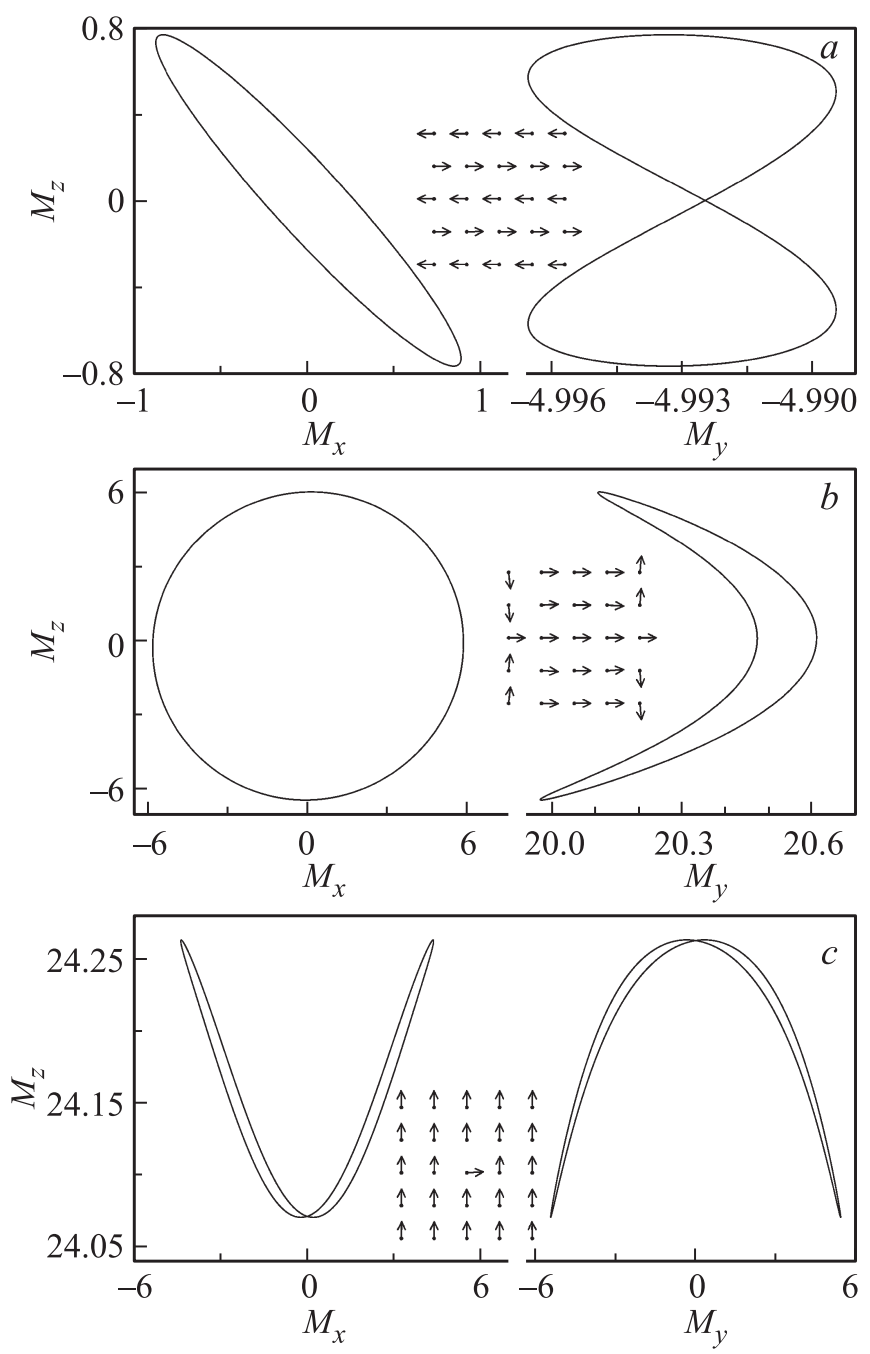

Рис. 5. Проекции траекторий регулярных колебательных режимов суммарного магнитного момента решеток с $k_{1}=-10$ при действии переменного магнитного поля, линейно поляризованного вдоль оси $X$ с амплитудой $h_{0 x}=1$ и частотой $\Omega=20$, и исходные конфигурации системы.
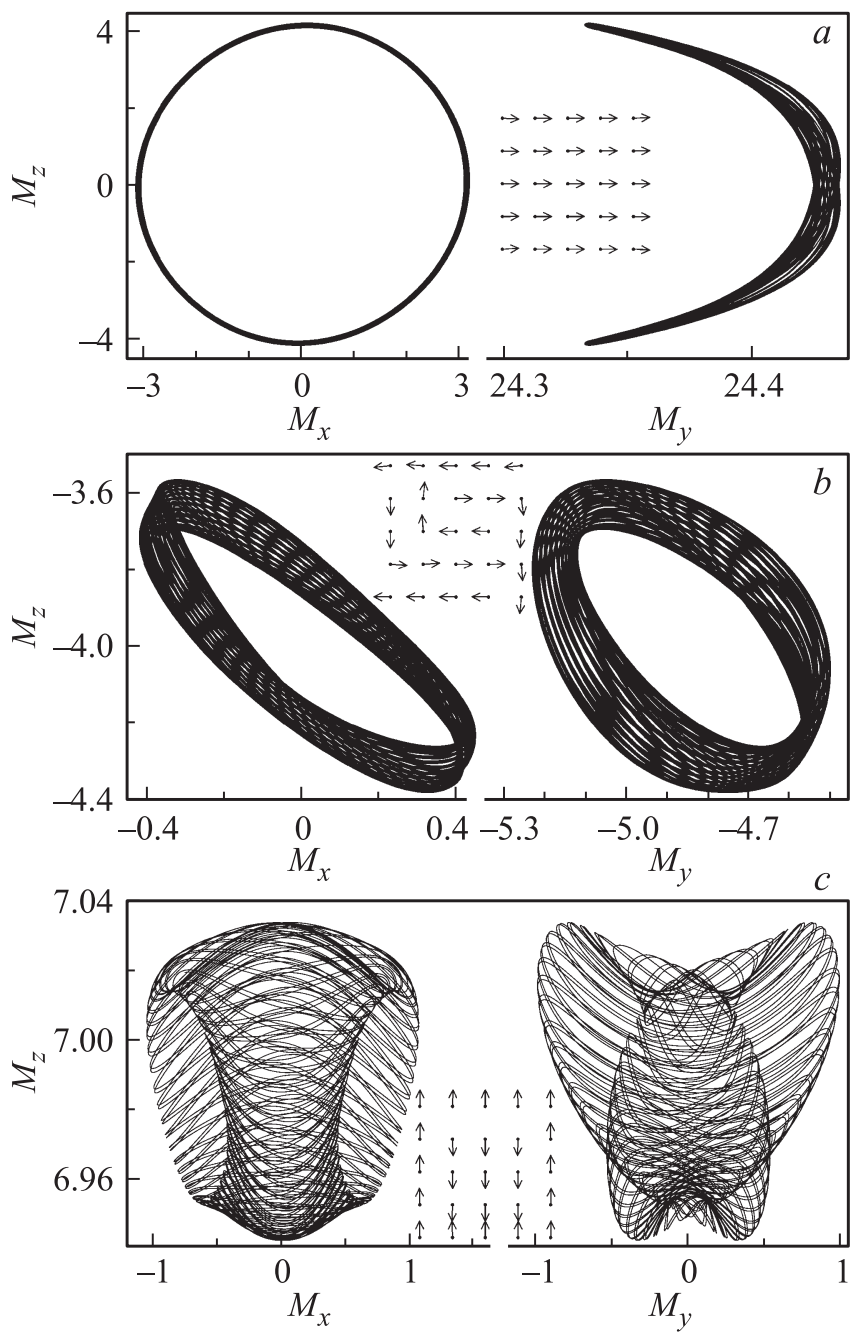

Рис. 6. Проекции траекторий стохастического $(a)$ и квазирегулярных $(b, c)$ колебательных режимов суммарного магнитного момента решеток с $k_{1}=-10$ при действии переменного поля, линейно поляризованного вдоль оси $X$ с $h_{0 x}=1$ и $\Omega=20$, и исходные конфигурации системы.

здесь и далее принят равным $\alpha=0.01$. Кривая 1 отвечает случаю, когда „подняты“ магнитные моменты граничащие друг с другом и лежащие на диагонали в центре диполей; кривые 2 - двум диполям центрального ряда, разделенных одним центральным диполем; кривые 3 двум крайним диполям одной диагонали. Видно, что при первой конфигурации устанавливаются колебания с наибольшей амплитудой, при второй конфигурации колебания магнитного момента близки к линейно поляризованным вдоль оси $X$, а при третьей конфигурации колебания оказываются хаотическими.

Далее рассмотрим колебательные режимы, вызванные переменным магнитным полем, при различной исходной конфигурации дипольной структуры, в случае, когда все магнитные моменты лежат в плоскости системы. На рис. 5 и 6 приведены исходные конфигурации решеток с константой анизотропии $k_{1}=-10$ и проекции траекторий колебательных режимов суммарного магнитного 

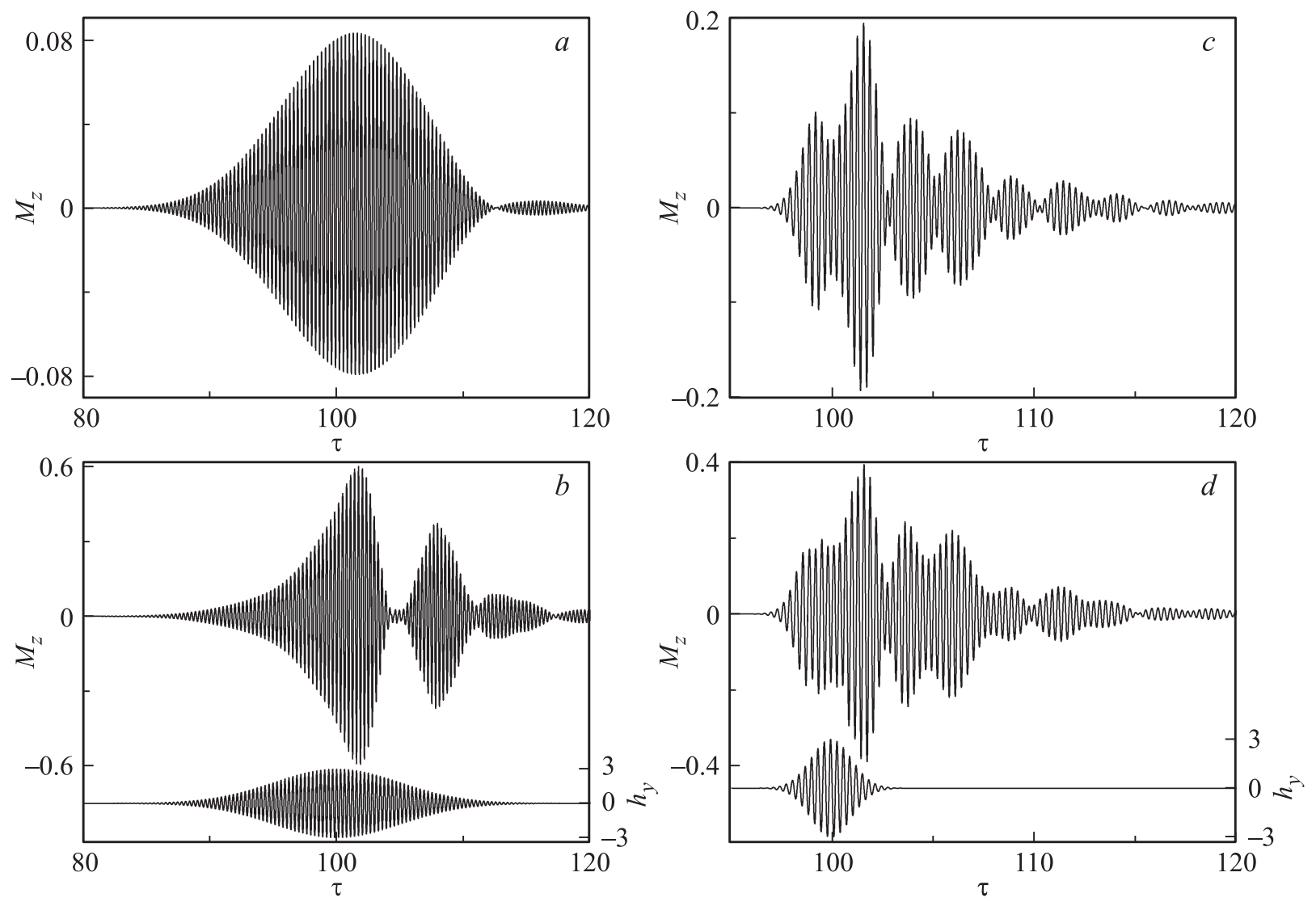

Рис. 7. Зависимость от времени $z$-компоненты магнитного момента системы с $k_{1}=-10$ при „поднятом“ центральном диполе и действии импульса линейно поляризованного вдоль оси $Y$ поля с $h_{0 y}=1(a, c), h_{0 y}=3(b, d), \tau_{0}=5(a, b), \tau_{0}=1(c, d)$; частота $\Omega=20$; зависимость $h_{y}(\tau)$ для $h_{0 y}=3(b, c)$.

момента при действии переменного магнитного поля. Поле линейно поляризованно вдоль перпендикулярной к плоскости систем оси $X$ имеет амплитуду $h_{0 x}=1$ и частоту $\Omega=20$. В случае рис. 5 устанавливаются регулярные колебательные режимы магнитного момента системы. При этом в случае $(a)$ возникают колебания одной из плоскостных компонент магнитного момента на удвоенной относительно внешнего поля частоте. В случаях $(b)$ и $(c)$ реализуются колебательные режимы с осями $Y$ и $Z$, близкие к гармоническим. На рис. 6 имеет место режим со слабой стохастичностью $(a)$, а также квазирегулярный режим колебаний [16] с аттрактором в виде тора $(b)$ и квазирегулярный режим с более сложным аттрактором $(c)$. Во многих не представленных на рисунке случаях возникают стохастические режимы, по виду близкие к белому шуму. При выбранной амплитуде переменного поля после прекращения его действия, как правило, устанавливаются конфигурации решеток, отличные от исходных.

\section{5. Отклик дипольных систем на импульс магнитного поля}

Рассмотрим системы, в которых только один из магнитных моментов ориентирован вблизи нормали к плоскости решетки (остальные диполи ориентированы по оси $Y)$. Исследуем отклик данной системы на импульс внешнего магнитного поля, который имеет гауссову временную огибающую:

$$
\mathbf{h}(\tau)=\mathbf{h}_{0} \sin (\Omega \tau) \exp \left(-\tau^{2} / 2 \tau_{0}^{2}\right),
$$

где $\Omega$ - несущая частота, $\tau_{0}-$ длительность импульса. На рис. 7 показана зависимость от времени $z$-компоненты суммарного магнитного момента системы с $k_{1}=-10$ при „поднятом“ центральном диполе в случае воздействия на систему импульса линейно поляризованного вдоль оси $Y$ магнитного поля с параметрами $h_{0 y}=1(a, c), h_{0 y}=3(b, d), \tau_{0}=5(a, b)$, $\tau_{0}=1(c, d)$, частота $\Omega=20$. Зависимость $h_{y}(\tau)$ для $h_{0 y}=3$ приведена на рисунках $(b)$ и $(c)$. Видно, что увеличение амплитуды импульса приводит к увеличению амплитуды отклика системы, а также может усложнять его, разбивая отклик на несколько цугов $(b)$. При сужении импульса $(c, d)$ динамический отклик системы также разбивается на цуги и становится более длительным относительно времени действия импульса.

При достаточно больших амплитудах импульса может произойти перескок поднятого центрального диполя к симметричной относительно системы ориентации или его укладывание в плоскость решетки. Однако данные 

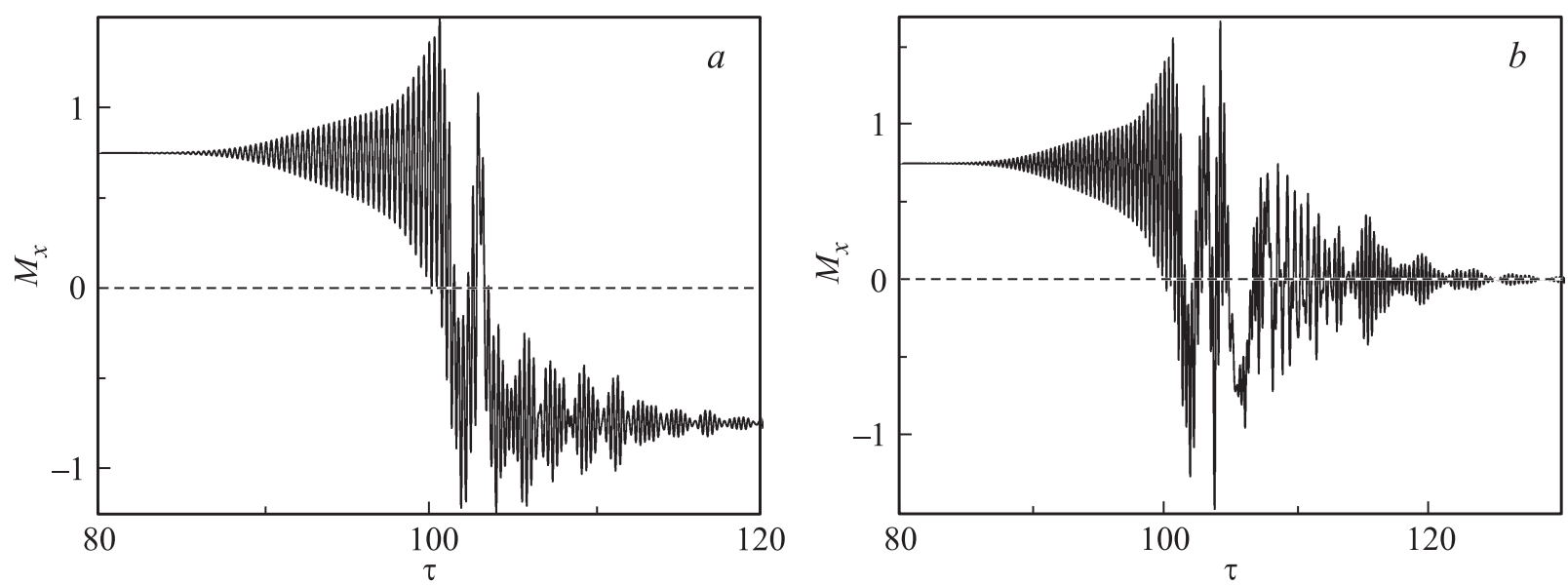

Рис. 8. Зависимость от времени $x$-компоненты магнитного момента системы, отвечающей рис. 7 , при действии импульса с параметрами $h_{0 y}=4,4.1(a, b)$ и $\tau_{0}=1$.
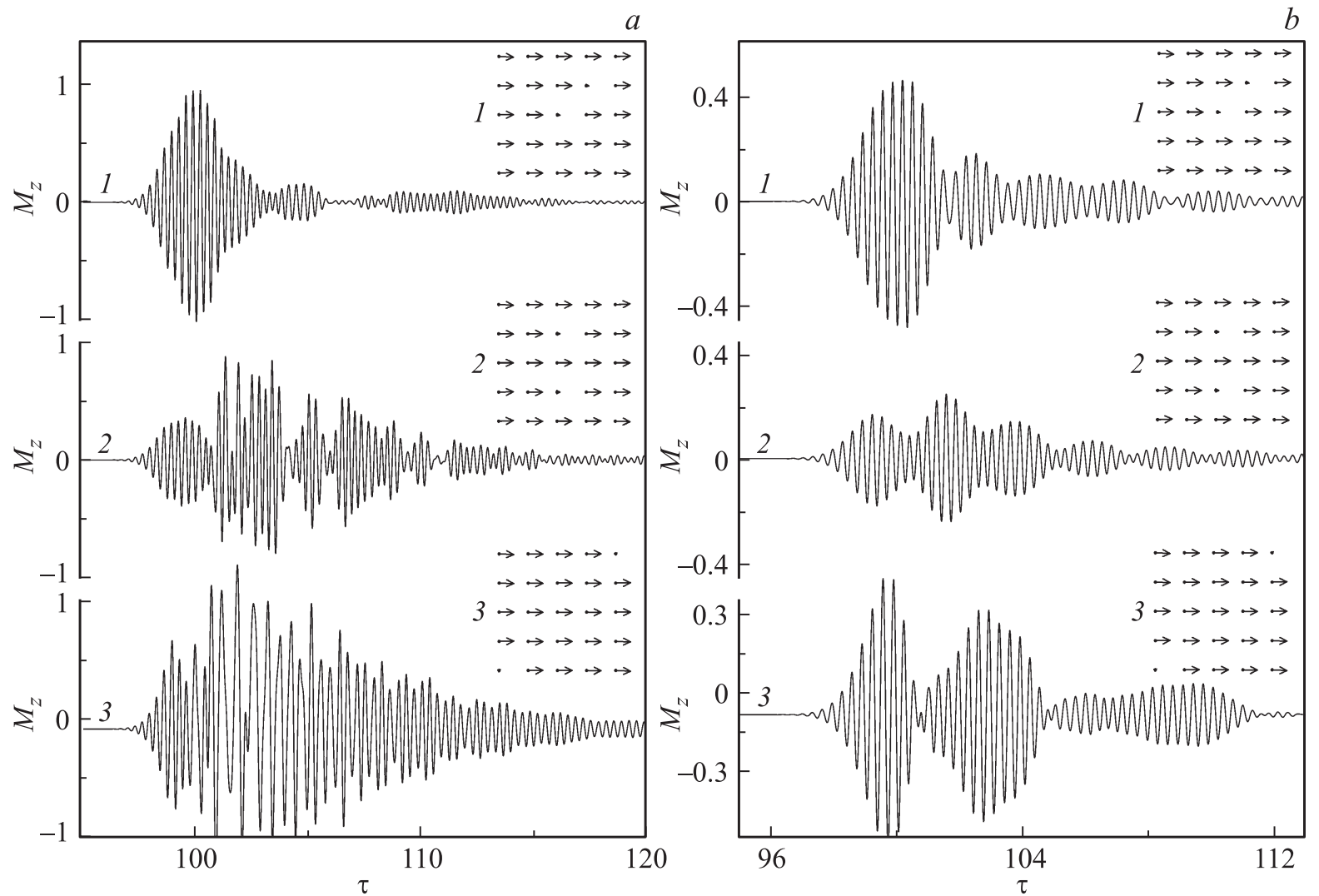

Рис. 9. Зависимость от времени $z$-компоненты магнитного момента решетки с $k_{1}=-10$ и приведенными конфигурациями при действии импульса с несущей частотой $\Omega=20$ и параметрами $h_{0 y}=3, \tau_{0}=5(a)$ и $h_{0 y}=1, \tau_{0}=1(b)$.

переходы носят случайный характер. Так, на рис. 8 приведена зависимость от времени $x$-компоненты магнитного момента вышерассмотренной системы при действии импульса с параметрами $\tau_{0}=1, h_{0 y}=4$ и $4.1(a, b)$. В первом случае $(a)$ после коротких хаотических колебаний происходит перескок центрального диполя в симметричное относительно плоскости положение, а во втором случае $(b)$ диполь укладывается в плоскость решетки.
Исследуем отклик на импульс магнитного поля рассмотренных выше решеток с двумя „поднятыми“ диполями. Для указанных систем на рис. 9 приведена зависимость от времени $z$-компоненты суммарного магнитного момента решетки с константой анизотропии $k_{1}=-10$ при действии импульса с несущей частотой $\Omega=20$ и параметрами $h_{0 y}=3, \tau_{5}=5$ (a) и $h_{0 y}=1, \tau_{0}=1(b)$. Видно, что наименьшие колебания магнитного момента возникают в случае второй конфигурации, а при увели- 


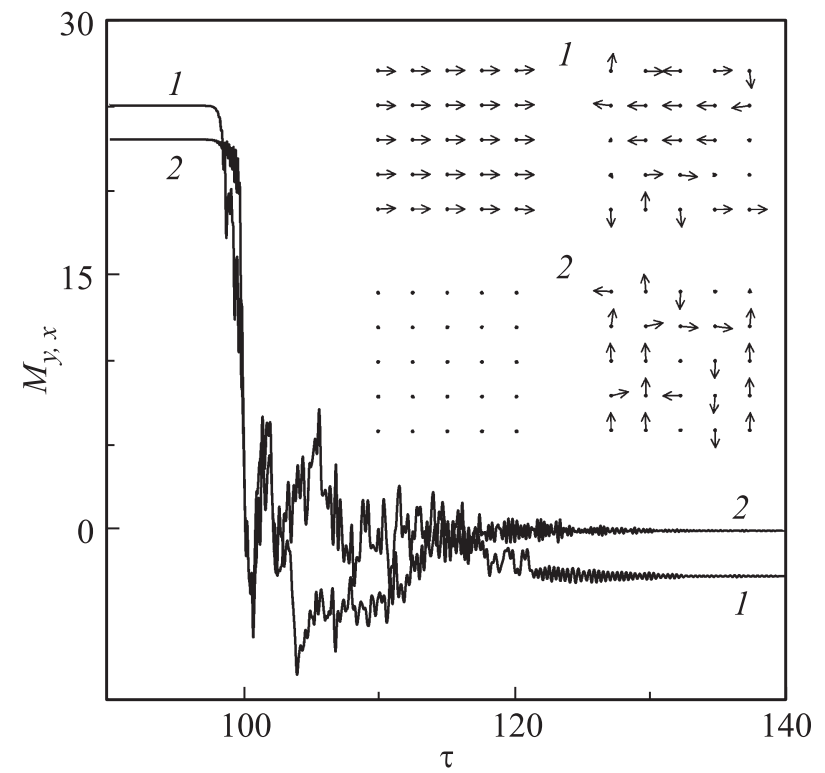

Рис. 10. Зависимость от времени компонент магнитного момента системы с $k_{1}=-10$ при ее переходе в другую ориентационную конфигурацию под действием импульса внешнего поля с параметрами $h_{0 x, y}=10, \tau_{0}=1, \Omega=20$; исходные и конечные конфигурации системы.

ченных продолжительности и амплитуде действующего импульса $(a)$ в случае второй и третьей конфигураций проявляются хаотические колебания, тогда как в случае первой конфигурации хаотизация отсутствует.

При действии сильных импульсов исходная равновесная конфигурация может перейти в одну из равновесных конфигураций, характеризуемых, как правило, меньшим значением энергии и большей энтропией. На рис. 10 приведена зависимость от времени суммарного магнитного момента системы с $k_{1}=-10$ при ее переходе в другую ориентационную конфигурацию под действием импульса внешнего поля с параметрами $h_{0 x, y}=10, \tau_{0}=1$, $\Omega=20$. В случае 1 исходной является конфигурация с магнитными моментами диполей, ориентированными по оси $Y$, поле импульса поляризовано вдоль оси $X$. В случае 2 исходной является перпендикулярная конфигурация, когда все диполи ориентированы по оси $X$, а поле импульса поляризовано вдоль оси $Y$. Переход от начальной конфигурации к конечной осуществляется после хаотических колебаний и носит случайный характер. В результате конечные ориентационные конфигурации также являются случайными. Однако в обоих случаях суммарный магнитный момент конечных конфигураций значительно меньше магнитного момента исходных конфигураций, при которых энтропия систем минимальна, так как диполи ориентированы в одном направлении.

Приведем переход от безразмерных величин к размерным для дипольной решетки из наночастиц железа: магнитный момент наночастицы $m \approx 3 \mu_{B} N$, где $N$ - число атомов в наночастице, ее размер $D \approx a(6 N / \pi)^{1 / 3}$, параметр кристаллической ре- шетки $a=0.286 \mathrm{~nm}$. Для $N=512$ ее размер составляет $D \approx 2.8 \mathrm{~nm}$, a $m \approx 1.4 \cdot 10^{-17} \mathrm{erg} / \mathrm{Oe}$. Для параметра дипольной решетки $d \approx 10 \mathrm{~nm}$ с учетом $\gamma=1.76 \cdot 10^{7}(\mathrm{Oe} \cdot \mathrm{s})^{-1}$ получаем следующие численные оценки для времени $t=\left(d^{3} / \gamma m\right) \tau \approx 4 \cdot 10^{-9} \tau \mathrm{s}$, частоты $\omega=\left(\gamma m / d^{3}\right) \Omega \approx 2.5 \cdot 10^{8} \Omega \mathrm{s}^{-1}$, магнитного поля $H=\left(m / d^{3}\right) h \approx 14.2$ Ое и константы анизотропии $K_{1}=\left(\mathrm{m}^{2} / V d^{3}\right) k_{1} \approx 1.75 k_{1} \cdot 10^{5} \mathrm{erg} / \mathrm{cm}^{3}$.

\section{6. Заключение}

Исследование дипольных решеток при наличии кубической анизотропии показало, что при константах анизотропии $\left|K_{1}\right|>6 m^{2} / V d^{3}$ или $\left|k_{1}\right|>6$ диполь-дипольное взаимодействие слабо влияет на ориентацию магнитных моментов диполей системы. В результате этого могут быть реализованы (в частности, с помощью локальных полей) различные равновесные конфигурации, при которых отдельные моменты ориентируются вдоль кристаллографических осей („легких“ намагничиваний). При этом каждая из ориентационных конфигураций характеризуется определенным откликом на слабые внешние импульсы магнитного поля, а также устанавливаемым стационарным колебательным режимом при действии внешнего переменного поля. Это дает возможность использовать подобные дипольные системы в качестве элементов хранения информации, в которых сильным внешним полем создается определенная равновесная конфигурация при получении в дальнейшем характерного отклика на слабый магнитный импульс или стационарное переменное поле.

Исследования проведены на примере квадратных решеток $5 \times 5$ с различной конфигурацией в плоскости системы, а также при ориентировании одного или двух диполей в направлении нормали. При этом показано, что отклик системы на внешний импульс зависит как от числа „поднятых“ диполей, так и от их взаимного расположения. Отношение времени отклика системы к длительности импульса увеличивается при сужении последнего. В случае плоскостных конфигураций - когда все диполи лежат в плоскости решетки - внешним переменным полем могут устанавливаться различные регулярные режимы колебаний, квазипериодические, а также хаотические режимы динамики суммарного магнитного момента решетки в зависимости от вида исходных конфигураций и параметров поля. В ряде случаев выявлены режимы с удвоенной относительно внешнего поля частотой колебаний одной из компонент магнитного момента. Показано, что в случае достаточно больших импульсов внешнего поля происходит изменение конфигурации системы, которое носит преимущественно случайный характер и приводит к увеличению энтропии системы. Полученные результаты носят общий характер и справедливы для различных решеток диполей с кубической анизотропией, которые, как показали проведенные исследования, могут быть использованы в качестве элементов хранения информации. 


\section{Список литературы}

[1] R. Skomski. J. Phys.: Condens. Matter. 15, R841 (2003).

[2] А.А. Фраерман. УФН 182, 1345 (2012).

[3] П.В. Бондаренко, А.Ю. Галкин, Б.А. Иванов. ЖЭТФ 139, 1127 (2011).

[4] С.А. Дзян, Б.А. Иванов. ЖЭТФ 142, 969 (2012).

[5] С.А. Дзян, Б.А. Иванов. ЖЭТФ 143, 1131 (2013).

[6] Ю.П. Иванов, А.И. Ильин, Е.В. Пустовалов, Л.А. Чеботкеич. ФТТ 52, 1576 (2010).

[7] В.А. Кособукин, Б.Б. Кричевцов. ФТТ 52, 759(2010).

[8] С.А. Гусев, Ю.Н. Ноздрин, М.В. Сапожников, А.А. Фраерман. УФН 170, 331 (2000).

[9] И.Р. Каретникова, И.М. Нефедов, М.В. Сапожников, А.А, Фраерман, И.А. Шерешевский. ФТТ 43, 2030 (2001).

[10] A.M. Shuty̌̌, S.V. Eliseeva, D.I. Sementsov. Phys. Rev. B 91, 024421 (2015).

[11] A.M. Shutyǐ, D.I. Sementsov. JMMM 401, 1033 (2016).

[12] А.М. Шутый. ЖЭТФ 145, 1048 (2014).

[13] А.М. Шутый, Д.И. Семенцов. Письма в ЖЭТФ 99, 806 (2014).

[14] Л.Н. Котов, Л.С. Носов, Ф.Ф. Асадуллин. ЖТФ 78, 60 (2008).

[15] А.Г. Гуревич, Г.А. Мелков. Магнитные колебания и волны. Физматлит, М. (1994). 464 с.

[16] А.Ю. Лоскутов, А.С. Михайлов. Основы теории сложных систем. НИЦ „РХД“, „ИКИ“, М.-Ижевск. (2007). 620 с. 